A LIVELY ELECTRONIC COMPENDIUM OF RESEARCH, NEWS, RESOURCES, AND OPINION

Astronomy Education Review

Volume 2, Sep 2003 - Jan 2004

Issue 2

\title{
First-Year College Students' Ideas About Astronomy: A Pilot Study
}

by Glenn Simonelli

Indiana University School of Education

Catherine A. Pilachowski

Indiana University Bloomington

Posted: 12/11/03

The Astronomy Education Review, Issue 2, Volume 2:166-171, 2004

(C) 2003, Glenn Simonelli. Copyright assigned to the Association of Universities for Research in Astronomy, Inc.

We have conducted pilot study of 148 first-year college students' ideas about astronomy. The purpose of this study is to gain insight into the students' understanding of astronomy. Specifically, the study was planned to answer two questions:

- What basic astronomical concepts do first-year college students have?

- What misconceptions about the Universe do first-year college students possess?

The students were given a brief survey containing four open-ended questions. The survey questions were formulated to elicit information providing insight into the following five research interests:

1. What do first-year college students know about the relative scale of objects in the Universe, and the arrangement and abundance of those objects?

2. How aware are first-year college students of the many different objects found in the solar system?

3. What do first-year college students know about the origin of the solar system?

4. How aware are first-year college students of the differences between the size, state, age, and surfaces of stars and planets?

5. How aware are first-year college students of the conditions needed to support life, the difficulties of discovering life if it exists (evidence needed to confirm, distances to life-supporting objects), and the difference between life in general and advanced life?

Our goal was to provide an opportunity for students to display the depth and range of their astronomical understanding and to reveal misconceptions and areas of uncertainty. Although the surveys do not reflect the totality of understanding on any one particular student--or even provide an accurate portrayal of the astronomical knowledge held by a "typical" college freshman-level student--taken collectively, they 
provide insight into the spectrum of understanding that students are likely to bring to a freshman-level astronomy class.

The four questions included in the survey were:

1. What do you think is the difference between a solar system and a galaxy? (This question was designed to explore students' ideas about the scale, arrangements, and abundance of objects in the Universe, as well as their awareness of objects in the solar system.)

2. How do you think our solar system was formed? Explain what you think happened. (This question was intended to explore students' knowledge about the origin of the solar system.)

3. How is the Sun different from our planet? (This question was designed to probe for information about the size, state, age, and surface of stars and planets.)

4. Do you think we will ever discover life on other planets? Why or why not? (This sought references to conditions needed to support life and the difficulties of discovering life if it exists.)

To ensure content validity, the survey was developed in consultation with two additional education professors and a second astronomy professor. Questions were written by the researcher and sent to the consultants for comments. Comments were incorporated into question revisions, and this process was repeated several times until the four final questions were selected.

The survey questions were distributed to the approximately 250 students in Dr. Pilachowski's freshman-level astronomy class, A105: Stars and Galaxies. It can be assumed that the students in the class were not declared astronomy majors. The class is designed for the general population of college students. It cannot be used for credit toward an undergraduate degree in astronomy but does satisfy a general science requirement. Several students in the class reported having taken a previous astronomy class called A100: The Solar System. It is assumed that students who took this class would have a greater knowledge of the solar system than the general class population. However, these students generally make up a small minority of the students in this class. It is reasonable to assume that the great majority of the students taking A105 have not had any previous college-level astronomy classes.

Of the four survey questions, question 2 proved to be the most enlightening, particularly in terms of illuminating misconceptions. Comments made by the professor and by several students prior to the administration of the survey addressed topics related to questions 1 and 3 , and it is currently not possible to determine if the responses to these questions reflect students' prior knowledge or information gained during the discussion preceding the survey. Question 4 was not written specifically to elicit pre/misconceptions; the speculative nature of the question did not require specific factual assertions by the responders. Although all four questions yielded interesting data that invite further investigation, the responses to question 2 were particularly interesting.

In general, there were seven main categories that the responses to this question could be placed in: accretion, Big Bang, capture, congregation, divine creation, explosion, and other, with many explanations containing elements of two or three different categories. The categories are listed below, with the number of responses in that category in parentheses. Explanations that include elements of more than one category are also listed and tallied separately. 
- Accretion (25)

- Accretion/Big Bang (4)

- Accretion/the Big Bang/capture (1)

- Accretion/capture (8)

- Accretion/congregation (2)

- Accretion/explosions (4)

- Big Bang theory (30)

- Big Bang/capture (2)

- Capture by gravity (11)

- Capture by magnetism (2)

- Capture by magnetism/explosion (1)

- Congregation (7)

- Divine creation (11)

- Divine creation/explosion (1)

- Explosion (10)

- Exploding stars or supernovae (6)

- Other explanations/difficult to classify (7)

Including multiple category explanations with all main categories mentioned yields the following results:

- Accretion (44)

- Big Bang (37)

- Capture (25)

- Explosion (22)

- Divine creation (12)

- Congregation (9)

- Other (7)

Eleven responders wrote that they were unsure. Five subjects left this question blank.

\section{Accretion}

Responses that describe a process of smaller particles coming together to form larger objects make up this category. These are distinguished from the capture and the congregation responses mainly by the responders' description of the size of the particles that came together. Of these responses, twelve mentioned gases or gas clouds, four mentioned dust or dust clouds, six mentioned both gas and dust as the building blocks, and one said that the planets were formed from materials left after the formation of the Sun. Two responders described the building materials as part of a nebula, and two mentioned planetesimals as part of the accretion process. Of the six responses that referred to the importance of temperature in the process, four specifically stated that cooling was part of the planet-formation process. There were no responses that used the word "accretion" in their explanations.

These explanations ranged from naïve to relatively sophisticated. Although accretion is the currently accepted explanation of the formation of the solar system, there were many inaccuracies and misconceptions expressed in these explanations. If one assumes that a complete explanation would discuss the Sun forming from a rotating disk of gas and dust and the planets forming after that from materials left 
in the disk, then there were no explanations that would be considered complete.

\section{Big Bang, Explosions, and Divine Creation}

The next most commonly offered explanations were responses that assumed that the Big Bang theory described the formation of the solar system. These results are consistent with those of Prather, Slater, \& Offerdahl (2002), which found that approximately $25 \%$ of college students interviewed thought that the Big Bang theory described the creation of planetary systems (as opposed to the nebular theory). Of the 37 inaccurate mentions of the Big Bang, nine of these responses described it as a type of "explosion," a characterization that many astronomers would discourage. In addition to these 37 responses, there were five responses that, although describing the Big Bang as a separate event, implied that the solar system formed shortly after the Big Bang. Of these five, three described the Big Bang theory reasonably accurately. In addition to explanations mentioning the Big Bang by name, several responses mentioned explosions as the event responsible for the formation of the Universe.

There were 18 responses that listed the Big Bang and little else. In a way, these are similar to the five responders who wrote "God," or "Creation" and nothing else. All of these appear to assume that ascribing a label or name to the process describes the actual process. It is worth noting here that, especially in the past, science education often emphasized labeling over process. It is hoped that this trend is changing.

Several of the explosion explanations seemed to use the term to refer to the formation of the Universe, and several others offered no explanation other than explosion, but there were several that specifically mentioned an exploding star as the source of materials from which the solar system was constructed.

\section{Capture and Congregation}

It is not always easy to distinguish explanations in these two categories from accretion explanations or from each other. In general, the explanations labeled capture tend to refer to larger objects being pulled into orbits around a gravity or magnetism source, whereas accretion explanations describe smaller particles forming larger ones. Both capture and congregation refer to objects moving into orbital positions, but capture explanations explicitly refer to a centralized source of attraction, whereas congregation explanations just refer to objects coming together.

Slightly under one-third of the students responding to the survey described the formation of the solar system as an accretion process. Gravity was accurately described as an attractive force in 25 responses. There were 25 responses that mentioned both a star/Sun and planets as components of a solar system. Of these 25 responses, nine explicitly mentioned that the Sun was formed first and the planets followed.

There were many weaknesses and misconceptions revealed, the most common being the 37 responses asserting that the solar system formed during the Big Bang. Of the 148 students who responded, 130 gave explanations that were essentially inaccurate in one or more key elements. In addition to the inaccuracies already discussed, several important misconceptions occurred repeatedly.

Twenty-one responses suggested that the materials that formed the Sun and the planets came from different sources. Also apparent was confusion about the scale and arrangement of different collections of objects in the Universe. There was noticeable confusion about the definitions of solar system, galaxy, and Universe, most notably in the nine congregation explanations. Another 10 responses showed some confusion about the nature of solids and gases, often having gases from stars forming solid planets. 
The broad range of accurate information related in the college students' answers--information about the Big Bang, supernovae, nebulae, and other astronomical phenomena--suggests that many students are receiving instruction about these topics sometime before the end of high school. However, virtually all of the college student responses showed some confusion about the formation process. This suggests that students entering college still do not have a clear mental model of the cosmos. Information about the Big Bang, solar nebulae, stellar and planet formation, supernovae, galaxies, and solar systems appears to be fragmentary and jumbled together in many of their minds. Although these students have a lot of information about different aspects of astronomy and cosmic history, most do not seem to have assembled these often-disparate pieces of information into a coherent, organized view of the cosmos.

In many public schools, Earth and space science is often taught for the last time in ninth grade. The breadth of information reflected in the responses to this question suggests that astronomy is covered in reasonable detail. However, the students' inability to retain this information in a way that presents a clear and logical view of the cosmos as they enter college is clearly revealed by their responses to this question. Perhaps it is unreasonable to expect them to develop an accurate view of the cosmos at such an early age, or perhaps astronomy is being taught in a way that leads to confusion. It is beyond the scope of this pilot study to answer that question.

There is reason to believe that most first-year college students are capable of thinking and learning about complex issues. The data, however, reveal several key areas of weakness in the students' conceptions of the Universe. Many surveys suggested that the authors did not have a clear understanding of the formation of the solar system, including a large number who confused the birth of the Universe with the beginnings of our solar system. Much of this confusion appears to stem from two areas. First, there is still apparent confusion about the structure of the Universe, including students who confused the definitions of solar system, galaxy, and Universe. Second, and probably more common, many responses suggested a misunderstanding of cosmic history. It is reasonable to assume that knowing that the Big Bang occurred 13.7 billion years ago and that our solar system formed nine billion years later would help separate these events in the minds of many students. Yet, the way this information is presented may have a profound impact on how well students understand the differences between these two events.

It is obvious that the students taking this survey have learned many facts about the Universe, but an ability to relate those facts to the overall picture is not apparent in the responses. The results of this survey suggest that it is not safe to assume that the goals of the Earth and space science areas of the National Science Standards--high school graduates with an accurate understanding of cosmic history and Earth's place in the Universe--have been consistently achieved thus far.

Although only a pilot study, the results invite further research. In particular, we would welcome attempts to repeat this survey with the additional collection of demographic information, allowing the results to be disaggregated accordingly. In addition, it would be interesting to conduct follow-up interviews to many of these responses. Finally, because this was, in essence, a qualitative rather than a quantitative methodology, some way of validating the researchers' interpretation of the data would be appropriate. 


\section{References}

Prather, E., Slater, T., \& Offerdahl, E. 2002, Hints of a Fundamental Misconception in Cosmology, Astronomy Education Review, 1(2), 28.

FR

$166-171$ 